\title{
Control of both particle and pore size in nanoporous palladium alloy powders
}

\author{
Christopher G. Jones, Patrick J. Cappillino, Vitalie Stavila, and David B. Robinson*
}

\begin{abstract}
Energy storage materials often involve chemical reactions with bulk solids. Porosity within the solids can enhance reaction rates. The porosity can be either within or between individual particles of the material. Greater control of the size and uniformity of both types of pore should lead to enhancements of charging and discharging rates in energy storage systems. To control both particle and pore size in nanoporous palladium (Pd)-based hydrogen storage materials, we have first created uniformly sized copper particles of about $1 \mu \mathrm{m}$ diameter by reduction of copper sulfate with ascorbic acid. In turn, these were used as reducing agents for tetrachloropalladate in the presence of a block copolymer surfactant. The copper reductant particles are geometrically self-limiting, so the resulting Pd particles are of similar size. The surfactant induces formation of $10 \mathrm{~nm}$-scale pores within the particles. Some residual copper is alloyed with the Pd, reducing hydrogen storage capacity; use of a more reactive Pd salt can mitigate this. The reaction is conveniently performed in gram-scale batches.
\end{abstract}

Keywords: mesoporous, monodisperse, uniform, hydride, microspheres, copper

\section{Introduction}

Hydrogen fuel cell technology is among the most promising alternative energy systems being developed to replace fossil fuels. Metal hydride batteries are frequently used in electric vehicles due to their safety and reliability [1]. The power densities of both of these technologies are limited by rates of chemical reactions at electrode surfaces, and their lifetimes are often diminished by changes in electrode geometry [2]. Efficiency and range are impacted if a battery cannot absorb braking energy rapidly enough in an electric vehicle [3]. Nanoporous palladium can provide fast charging and discharging in hydrogen gas storage systems, and accommodate swelling and shrinking of electrodes [4]. While the cost of Pd may be prohibitive for many applications, study of this material may lead to increased efficiency, reliability, and range of the more general class of hydrogen-based energy storage and conversion devices. A convenient, inexpensive and scalable method to produce nanoporous Pd with control of both nanopore size and particle size would ease further research and development of clean energy technology and help reduce our overall dependency on fossil fuel. 
Pd in particular has a number of advantageous properties that make it a prime choice for the energy technologies discussed above. Pd has the ability to absorb up to 900 times its volume of hydrogen at room temperature and pressure, and then release it with only mild heating or pressure reduction $[5,6]$. The rate of uptake and release is inherently fast, but is often limited by such factors as surface area, particle size, and packing density. We seek to optimize these properties in order to improve upon current energy storage and conversion technologies that rely on the chemistry of hydrogen.

Nanoporous noble metals with highly uniform, ordered pores are usually made by reduction of metal salts in the presence of a surfactant [7]. The surfactant is at high enough concentrations that it forms arrays of cylindrical (or more complex) micelles a few nanometers in diameter, around which the metal is grown. The surfactant is then washed away. The metal can be reduced by electrodeposition on an electrode [8], use of a solution-phase reducing agent like ascorbic acid [9], a gas-phase reducing agent like hydrogen [10], or a solid-phase reductant like zinc metal [11]. The surfactant usually contains a poly(ethylene oxide) hydrophilic end. The hydrophobic end can be a long-chain alkyl group such as hexadecyl, or a hydrophobic polymer such as polystyrene. Adjusting the surfactant size allows tuning of the resulting pore size [4].

While these methods can reliably produce highly regular pores, there is much less control over largerscale geometry. An example from previous work in our laboratory is shown in Figure 1. In this case, uniform, regularly spaced nanopores are apparent, but the material arranges into irregularly shaped flakes on the $10 \mu \mathrm{m}$ scale. Control of particle size is complicated by the transport rates of the metal salt and reducing agent, and the geometry of particles growing through the surfactant mixture, typically leading to highly heterogeneous products. Planar processing methods have been reported that control both particle and pore size, but they require many steps and generate only about a milligram per batch [12,13]. We have developed a scalable strategy to control both the particle size and pore size in nanoporous Pd powders. We first create uniform-sized, nonporous particles of copper, a metal that is less noble than Pd. We then use these as self-limiting reducing agents for a palladium salt. The Pd should react 1:1 with the copper, resulting in a similarly sized porous Pd particle and a copper salt. This galvanic exchange reaction has been previously used in the production of nanoparticles and nanowires $[14,15]$ and in growth of atomic multilayer films [16]. Some of the copper remains in the form of an alloy that retains useful hydrogen storage properties [15]. By this approach, we can consistently produce micrometer-scale Pd alloy particles of uniform shape and size. Our work contrasts with the prior work on nanowires and films $[15,16]$ because it involves larger scale particles in larger scale batches. We expect to be able to adjust the particle and pore size by changing the surfactant or the reductant particle size. By adopting a literature procedure that uses inexpensive, dilute surfactants [17], we are able to routinely synthesize batch sizes 
large enough for many important characterization methods, enabling further research and development of these materials.
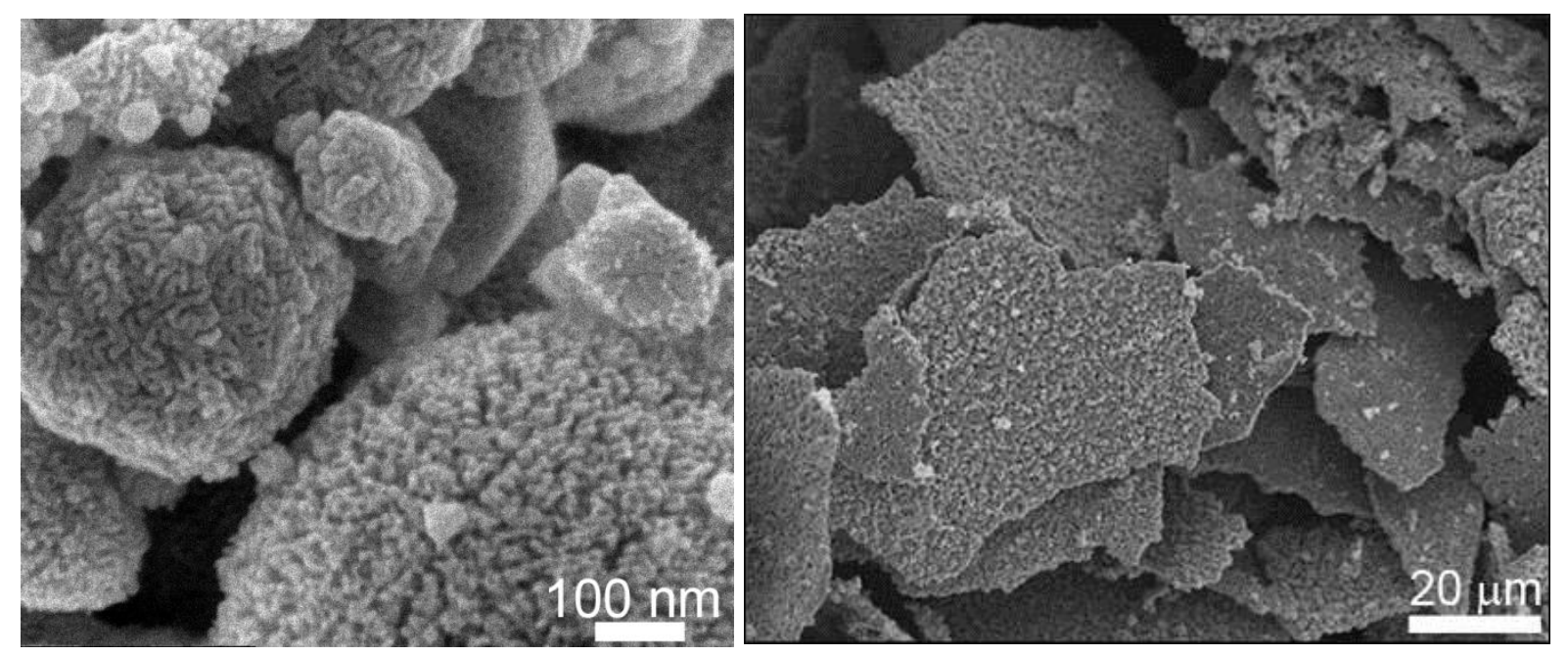

Figure 1: Nanoporous Pd reduced by $\mathrm{H}_{2}$ gas in a block copolymer template, produced according to Cappillino et al. [4], shows highly ordered pores (left) but uncontrolled particle shape and size (right).

\section{Experimental}

\subsection{Materials}

Copper(II) sulfate $\left(\mathrm{CuSO}_{4} \cdot 5 \mathrm{H}_{2} \mathrm{O}\right)$ was obtained from EM Science. Acetone, sodium chloride $(\mathrm{NaCl})$, sodium ascorbate $(>98 \%)$ and palladium nitrate dihydrate $\left(\mathrm{Pd}\left(\mathrm{NO}_{3}\right)_{2} \cdot 2 \mathrm{H}_{2} \mathrm{O}\right)$ were obtained from Aldrich. Ammonium tetrachloropalladate(II) $\left(\mathrm{H}_{8} \mathrm{Cl}_{4} \mathrm{~N}_{2} \mathrm{Pd}\right.$, 99.998\%) was obtained from Alfa Aesar. Pluronic F127 Prill is a low-cost triblock copolymer from BASF with a central block of hydrophobic poly(propylene oxide), with a degree of polymerization of about 56, and outer blocks of hydrophilic poly(ethylene oxide) each with a degree of polymerization of about 101, containing about $0.01 \mathrm{wt} \%$ 2,6-Di-tert-butyl-4methylphenol as an antioxidant. Deionized water was 18 megohm-cm and prepared in the laboratory.

\subsection{Synthesis of $\mathrm{Cu}$ particles}

A solution of copper sulfate $(6 \mathrm{~mL}, 1 \mathrm{M})$ was added to a solution of sodium ascorbate $(10 \mathrm{~mL}, 2 \mathrm{M})$ at room temperature. After approximately 2 minutes, the solution began to change from a dark brown to an orange/yellow color. Within 2-4 minutes when a yellow color had fully developed throughout the solution, $1 \mathrm{~mL} 10 \mathrm{wt} \%$ Pluronic F127 was added to the reaction. The addition of a surfactant did not result in pores in the copper, but prevents aggregation of the particles. This process does not require any stirring or other sustained agitation during the reaction period. 
To ensure adequate reaction time, the solution was left for 24 hours. After this, a noticeable copper precipitate had formed on the bottom of the vial. The copper and remaining solution were placed in a centrifuge tube for washing and extraction. The copper was first centrifuged at 8000 RPM for 2 minutes. The supernatant was then removed, and the copper was re-suspended in $1.0 \mathrm{wt} \%$ aqueous Pluronic F127, and again placed in the centrifuge for 2 minutes at 8000 RPM. This process was repeated 3 times in order to thoroughly remove any remaining reactants from the suspended copper solution. After the third wash, the copper was again suspended in $100 \mathrm{~mL} 1.0 \mathrm{wt} \% \mathrm{~F} 127$ for use as a reducing agent. The particles were used in subsequent steps immediately, or within a few days, being stored in a sealed container. Before storing, nitrogen was bubbled through the solution of suspended particles to prevent oxidation.

\subsection{Synthesis of Pd particles}

To prepare the palladium reactant solution, $1.0 \mathrm{wt} \%$ Pluronic F127 surfactant was dissolved in an aqueous sodium chloride solution $(135 \mathrm{~mL}, 3.5 \mathrm{M})$. After the surfactant had been dissolved, $\left(\mathrm{NH}_{4}\right)_{2} \mathrm{PdCl}_{4}(1.34 \mathrm{~g}$, $4.71 \mathrm{mmol}$ ) was added to the solution and lightly stirred until fully dissolved. The $100 \mathrm{~mL}$ of suspended $\mathrm{Cu}$ particles prepared above $(299 \mathrm{~g}, 4.71 \mathrm{mmol} \mathrm{Cu})$ was slowly added to the Pd solution with continuous stirring. This resulted in a gradual change of color from light brown to black over the course of approximately 10 minutes. Prior to this mixing step, nitrogen was bubbled through the suspension of $\mathrm{Cu}$ particles and the Pd salt solution to remove oxygen and reduce the rate of oxidation during the reaction period. The mixture was then left stirring while nitrogen was once again bubbled through. After another 10 minutes, the nitrogen line was removed and the flask was capped immediately. The flask contents were stirred for 48 hours. At this point, the formation of a black precipitate was noticeable on the bottom of the flask. The reaction mixture was placed in the centrifuge at 4000 RPM for 3 minutes. The remaining supernatant was discarded. The powder was re-suspended in $30 \mathrm{~mL}$ of acetone, and was again centrifuged at 4000 RPM for 3 minutes to remove surfactant and other byproducts. The acetone wash cycle was repeated 3 times, followed by a water wash cycle using the same procedure. The powder was dried overnight under reduced pressure.

\subsection{Treatment to reduce copper content}

Samples of nanoporous $\mathrm{Pd}(50 \mathrm{mg}, 0.47 \mathrm{mmol})$ were added to two solutions of $\mathrm{Pd}\left(\mathrm{NO}_{3}\right)_{2}(10 \mathrm{~mL}, 11.8$ $\mathrm{mM}$, and $10 \mathrm{~mL}, 23.6 \mathrm{mM}$ ) and placed on an orbital shaker for 24 hours to remove residual copper. These treatments corresponded to a 1:2 and 2:1 molar ratio of $\mathrm{Pd}\left(\mathrm{NO}_{3}\right)_{2}$ to $\mathrm{Cu}$, respectively. The powder was washed three times by suspending in $30 \mathrm{~mL}$ deionized water, centrifuging at $4000 \mathrm{RPM}$, and discarding the supernatant. The powder was then dried overnight under vacuum. 


\subsection{Characterization}

Scanning Electron Microscopy (SEM) was used to analyze the copper and palladium powders. SEM analysis was performed using either a JEOL JSM 7600F field-emission electron microscope or a Hitachi S-4500 field-emission electron microscope. Powder samples were spread over carbon tape placed on a silicon wafer for analysis. Energy-dispersive X-ray spectroscopy (EDS) was also performed using the JEOL JSM 7600F field-emission electron microscope.

Porosimetry was conducted using a Micromeritics ASAP 2020 porosimeter. Using nitrogen at $77 \mathrm{~K}$ as the adsorbate, BET surface area and BJH pore size distribution were obtained for Pd particle samples [ $18,19,20]$. Pd samples were degassed for 15 hours at $50^{\circ} \mathrm{C}$ before performing the analysis. Hydrogen storage properties of Pd samples were also measured using this instrument. Prior to measurements, Pd samples were cleaned to remove surface oxide by exposing samples to 8 Torr hydrogen gas at $298 \mathrm{~K}$, then evacuating [4]. This cleaning process was repeated three times.

XRD data were collected with a PANalytical Empyrean X-ray diffractometer equipped with a PIXcel ${ }^{3 \mathrm{D}}$ detector and operated at $45 \mathrm{kV}$ and $40 \mathrm{kA}$ using $\mathrm{Cu} K \alpha$ radiation $(\lambda=1.5418 \AA)$. The patterns were collected in the $2 \theta$ range of 30 to $130^{\circ}$ with a step size of $0.026^{\circ}$. XRD reference patterns and structural refinements were performed using the software package HighScore Plus ${ }^{\circledR}$.

\section{Results and discussion}

The strategy presented herein is to use a locally constrained dose of reducing agent to produce particles of a uniform and controlled size. Metals less noble than palladium are potentially low cost reducing agents. The metals have a molar volume comparable to our desired product, so we expect to be able to straightforwardly tune the product particle size by choosing a similar reductant particle size. There are numerous published procedures for chemical production of uniform micrometer-scale particles $[21,22,23]$. We modified a literature procedure [24] to create micrometer-scale copper particles by a simple, minimally hazardous reaction. Nickel, iron and cobalt are other convenient candidate materials, but they can aggregate due to magnetic interactions, and nickel and cobalt are more toxic. Each case requires optimization to ensure that the reaction goes to completion, and that side reactions and byproduct formation are controlled. 


\subsection{Synthesis of uniform $\mathrm{Cu}$ particles}

The use of tunably sized copper particles as a reducing agent offers great flexibility. Parameter variation during $\mathrm{Cu}$ synthesis such as reaction temperature, concentration and addition of surfactant has been shown to affect $\mathrm{Cu}$ particle shape and size [24]. This provides the ability to tune $\mathrm{Cu}$ particle shape and size to desired specifications. In this work, uniform spherical $\mathrm{Cu}$ particles (Figure 2) were chosen to produce Pd spheres with a narrow size distribution that replicates the copper particles.

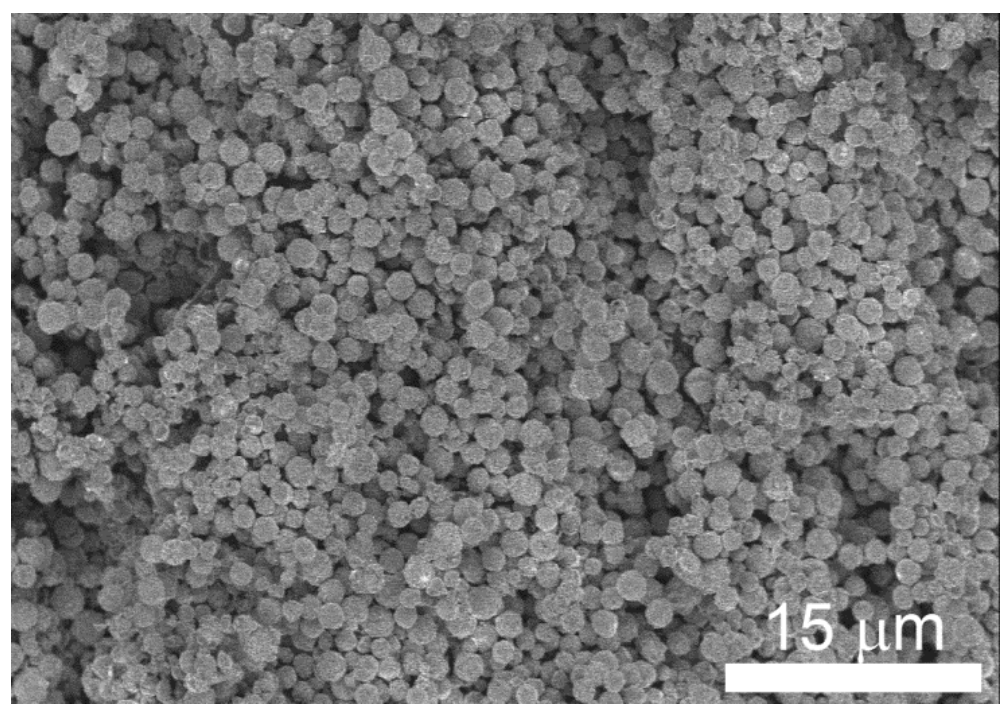

Figure 2: SEM image of copper particles synthesized using room temperature reaction of $\mathrm{CuSO}_{4}$ and sodium ascorbate

The copper reduction reaction appears to occur in two stages. When the solution of $\mathrm{CuSO}_{4}$ is mixed with sodium ascorbate, there is an initial color change to a yellow-colored, insoluble intermediate. The reaction then proceeds to form a dark red-brown product. A sample of the intermediate was isolated within the first two minutes of the reaction and analyzed using XRD to determine composition (Figure 3a). This initial reaction stage produces a $\mathrm{Cu}_{2} \mathrm{O}$ intermediate product shown by $\mathrm{SEM}$ in Figure 4. As the reaction goes to completion, the $\mathrm{Cu}(\mathrm{I})$ is further reduced, resulting in $\mathrm{Cu}$ metal as determined by XRD analysis (Figure 3b). 

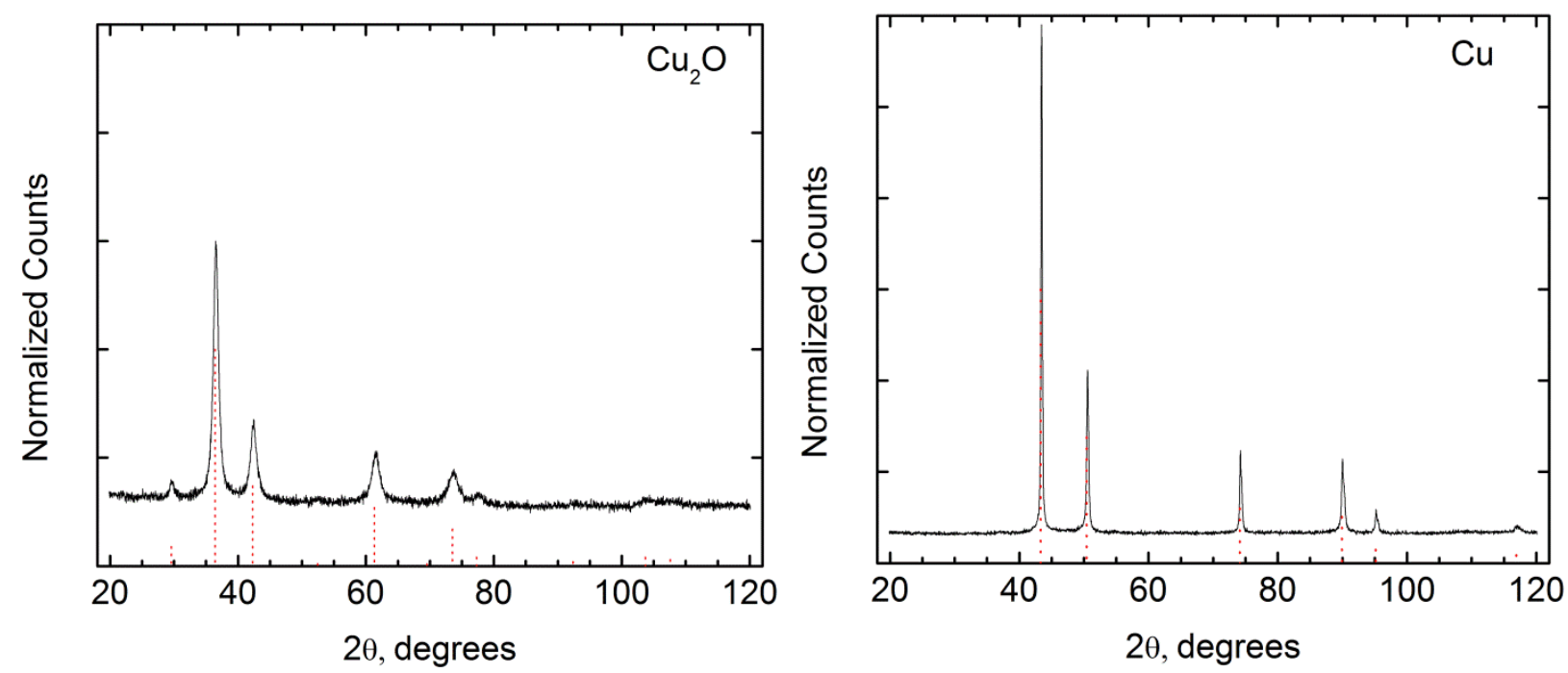

Figure 3: $\mathrm{Cu}_{2} \mathrm{O}$ was isolated mid-reaction (a), while pure copper metal is produced as the reaction goes to completion (b). Dashed lines represent reference peaks for the indicated materials.

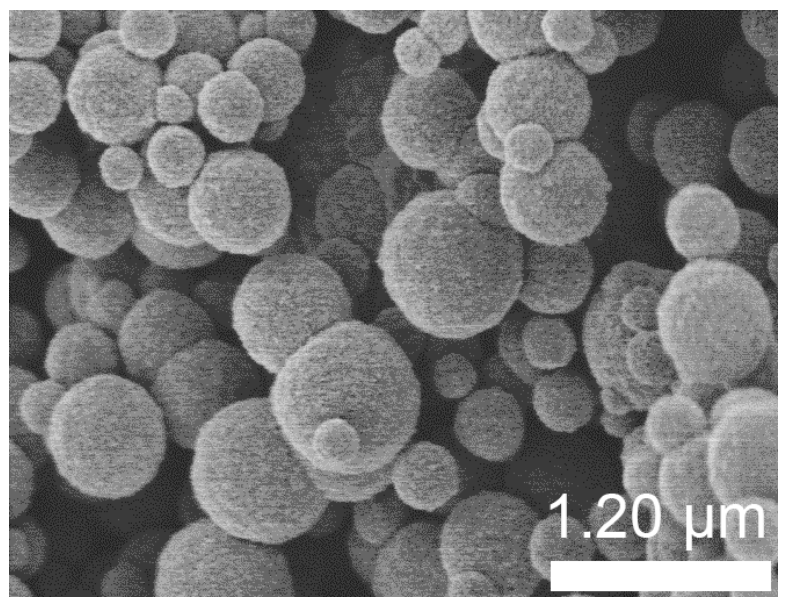

Figure 4: Intermediate $\mathrm{Cu}_{2} \mathrm{O}$ particles isolated within the first 2 minutes of reaction

Figure 4 reveals that although particle shape is similar to the final product, overall particle size is significantly smaller and appears to have a much wider distribution. Based on SEM imaging, $\mathrm{Cu}_{2} \mathrm{O}$ particle sizes range from $300 \mathrm{~nm}$ to $1 \mu \mathrm{m}$. Attempts to use the $\mathrm{Cu}_{2} \mathrm{O}$ particles as a reducing agent for $\mathrm{Pd}$ particle synthesis appeared to yield a Pd product, but not uniformity of particle or pore size.

For the strategy proposed herein to succeed, the copper particles must be isolated and in suspension. Inclusion of surfactant during the synthesis of $\mathrm{Cu}$ particles proved necessary to prevent aggregation and improve uniformity of size. To prevent aggregation, a small amount of surfactant was added shortly after mixing the $\mathrm{CuSO}_{4}$ and sodium ascorbate, in an attempt to solubilize the intermediate, but not interfere 
with its formation. Upon varying the timing of the addition of surfactant in the Cu reduction reaction, differences in the amount of aggregation were observed. Figure 5 shows SEM images of three samples of $\mathrm{Cu}$ particles in which the surfactant was added at various time points during the course of the reduction, and one sample without the addition of surfactant. Although the addition of surfactant is necessary to prevent excessive particle aggregation after reduction has occurred, the addition of surfactant too early was shown to reduce uniformity. When surfactant was added within the first 2 minutes after the start of the reaction, it was found to hinder the assembly of sub-micrometer particles into micrometer-scale spheres (figure 5a). Optimal uniformity was achieved when surfactant was added after the initial yellow color had dissipated, generally around 2-4 minutes after mixing at room temperature. It is likely at this point that the majority of the reduction had occurred, allowing the copper spheres to remain intact in the presence of the surfactant. The addition of surfactant after the initial 2-4 minute period had little to no effect on particle size or shape, only on the degree of agglomeration between spherical particles. Porosity in the $\mathrm{Cu}$ particles was not observed, regardless of when (or whether) surfactant was added. Although spherical particles have been formed without the use of a surfactant, this often causes aggregation during subsequent steps, which results in poor uniformity in the Pd reaction. This time window for surfactant addition allows ample time for particles to form during the initial reaction period while still remaining adequately suspended as necessary for Pd reduction. 

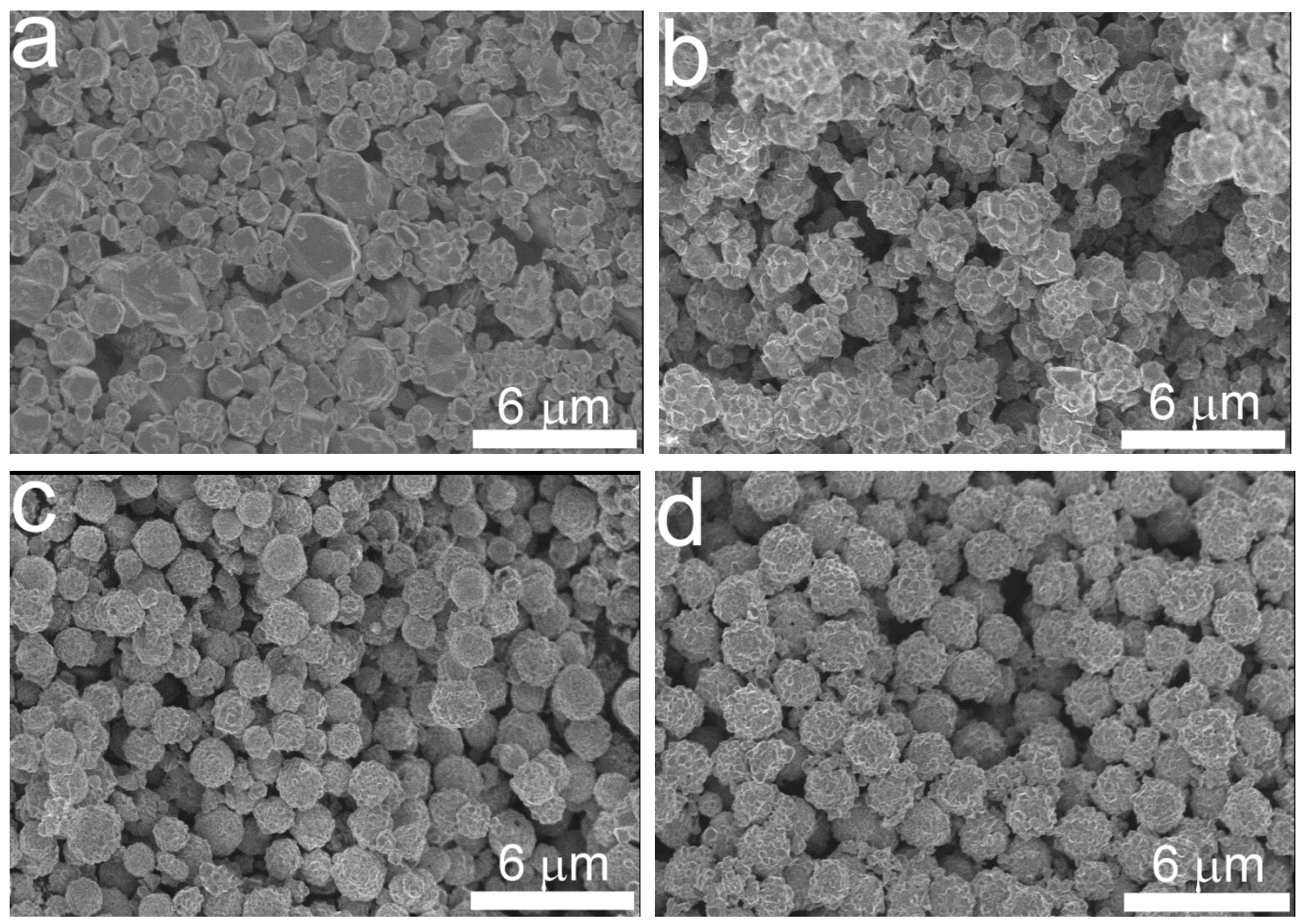

Figure 5: Cu particles with surfactant added at 1 minute (a), at 2 minutes (b), 3 minutes (c), and without (d).

\subsection{Synthesis of porous Pd particles}

To make porous $\mathrm{Pd}$ particles of the same size and shape as the initial $\mathrm{Cu}$ particles, the copper particles were added to an aqueous $\mathrm{Pd}$ salt in the presence of a surfactant, leading to pore formation. While much of the prior work on synthesis of porous metals uses concentrated aqueous surfactants, Wang et al. demonstrated that the dilute surfactants used herein can form pores when growth occurs at a predefined metal interface at a controlled rate [17]. The high viscosity of concentrated surfactants makes them inconvenient to prepare, sensitive to temperature, prone to entrainment of gas bubbles, and slow as a reaction medium due to the inhibition of convective mass transport [9]. The ability to use dilute surfactants thus confers a significant advantage.

Here, we use one of the surfactants studied by Wang et al., Pluronic F127, a low-cost triblock copolymer from BASF with hydrophobic poly(propylene oxide) as the middle block, between two hydrophilic poly(ethylene oxide) blocks [17]. This surfactant has been demonstrated to yield pores when electroplating a platinum film using $1.0 \mathrm{wt} \%$ surfactant solutions. Surfactant concentrations closer to 50 
wt $\%$ are typically used when making nanoporous metals. When dissolved, the F127 surfactant forms spherical micelles within the solution, according to the phase diagram by Wanka et al [25]. At electrode or particle surfaces, the effective concentration is probably higher due to adsorption of a surfactant layer onto the surface, and this may allow for formation of a 2D hexagonal or disordered close-packed structure on the surface. Pluronic F127 was shown to yield pores around $12 \mathrm{~nm}$ in diameter [17]. The surfactant template has limited effects on $\mathrm{Cu}$ particles due to the fact that it is added later in the reaction when the $\mathrm{Cu}$ is likely to be fully reduced.

Upon mixing, the $\mathrm{Cu}$ particles begin to reduce the Pd ions. As Pd ions are reduced, Pd metal forms within the surfactant template. Reduction reactions are carried out in the presence of a high concentration of $\mathrm{NaCl}$ to slow the reaction and reduce the likelihood of overgrowth or displacement of the surfactant. Reactions performed without excess $\mathrm{NaCl}$ showed unsatisfactory porosity and uniformity (Figure 6). Because $\mathrm{Pd}$ ions bind chloride more strongly than $\mathrm{Cu}$ ions, the presence of excess chloride in the reaction solution is expected to lower the reduction potential of Pd by Le Chatelier's principle, stabilizing the oxidized form of $\mathrm{Pd}$ more than that of $\mathrm{Cu}$, reducing the reaction overpotential, and slowing the chemical reduction process [16]. This provides a more controlled growth rate during the reaction, resulting in a more uniform particle distribution.

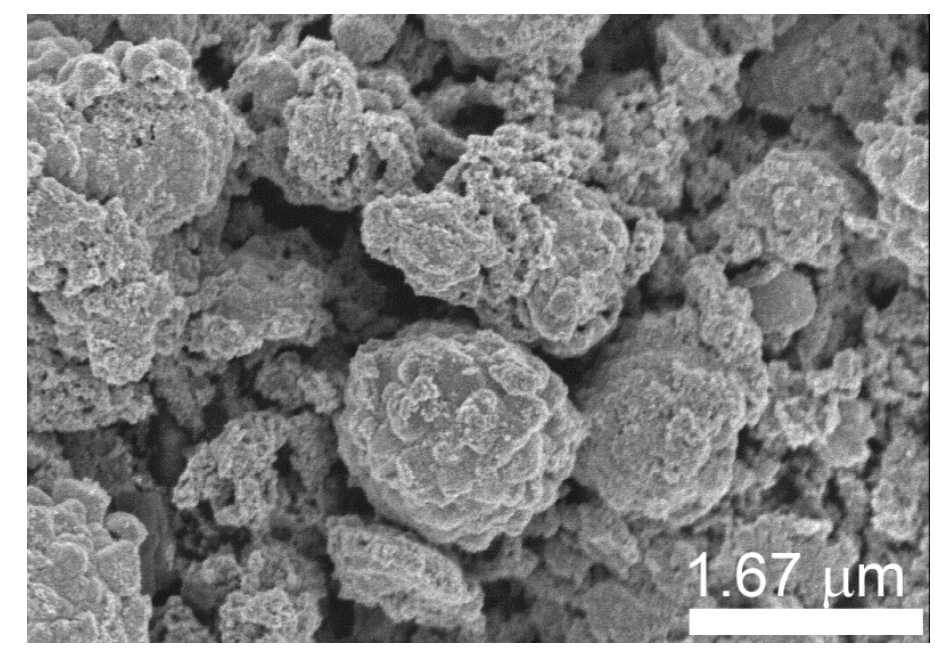

Figure 6: SEM image of Pd particles prepared using Cu particles in the absence of excess chloride.

With a slow and controlled growth rate, Pd metal forms within the surfactant template as it is reduced. After the Pd metal has been fully reduced, a washing process is necessary to remove the surfactant template from the synthesized particles. The particles were suspended in acetone, a solvent that readily dissolves the surfactant, and centrifuged in order to remove residual surfactant. Using a centrifuge speed 
of 4000 RPM has proven to be ideal due to the particles' tendency to deform at higher speeds. After a thorough wash process, the surfactant is removed, revealing a uniform-sized pore structure in its place.
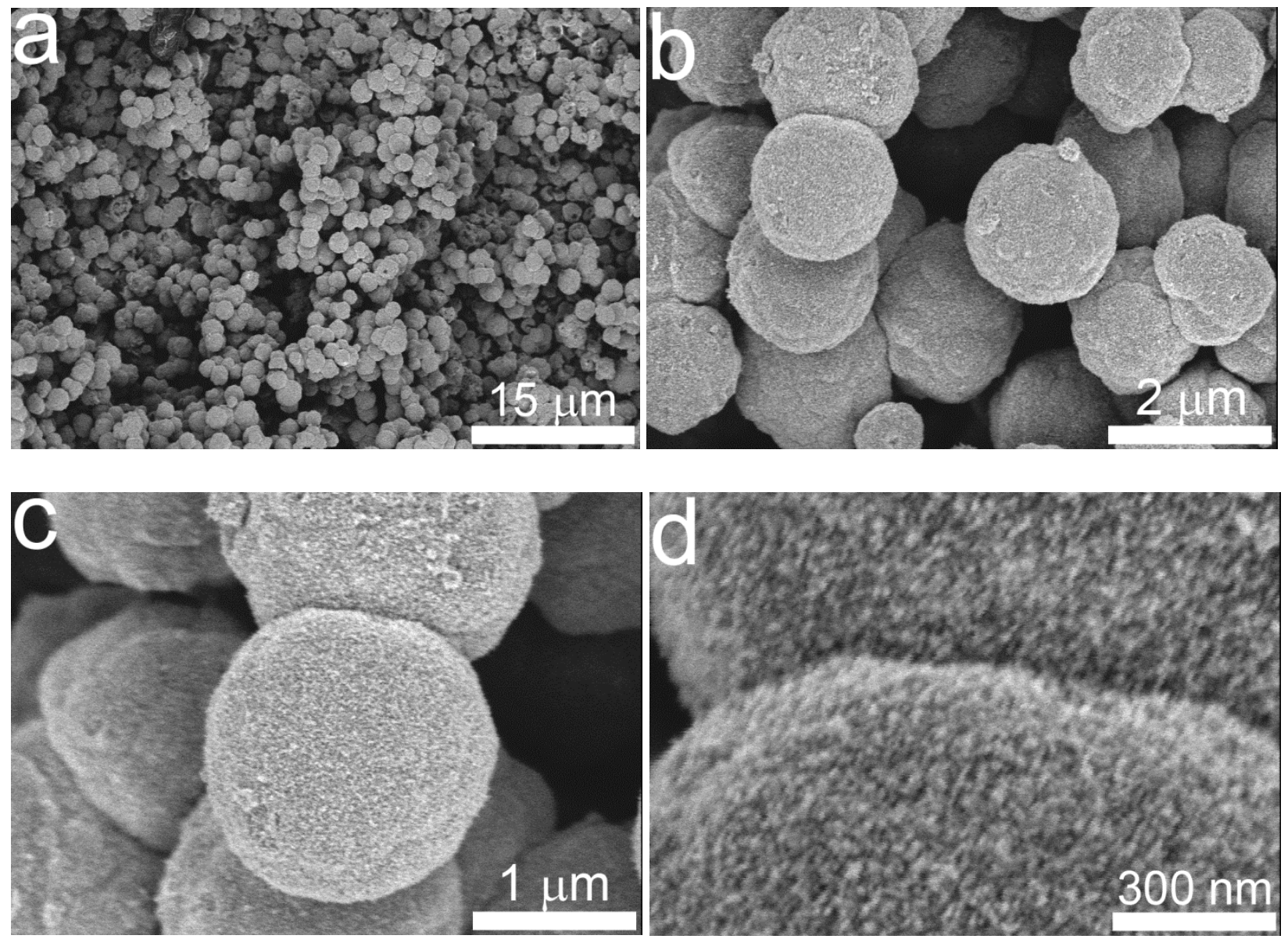

Figure 7: SEM images of Pd sample at x2.00k (a), x15.0k (b), x30.0k (c), x100k (d)

Physical characterization of the Pd particle morphology was conducted with SEM imaging after the chemical reduction process. Particle shape and size is observed to be consistent, with spherical particles of similar size throughout the sample. As shown in Figure 7, Pd particles with size and shape similar to the $\mathrm{Cu}$ particles used as reducing agents range from 1-2 $\mu \mathrm{m}$ in diameter. SEM images at higher magnification also revealed a highly porous surface (Figure 7d). Pore sizes range from approximately 10 $-20 \mathrm{~nm}$ in diameter and appeared uniform among particles.

The use of well suspended and uniform copper particles is critical to producing uniform size palladium particles (Figure 8a-b). Earlier attempts at creating uniform spherical palladium particles using less uniform or aggregated copper (either prepared without surfactant or when surfactant was added too early during the $\mathrm{Cu}$ reduction phase) produced similarly aggregated palladium with a relatively broad size and 
shape distribution (Figure $8 \mathrm{c}-\mathrm{d}$ ). The $\mathrm{Cu}$ preparation procedure described in the Experimental section instead reliably provides uniform $\mathrm{Cu}$ particles (Figure $8 \mathrm{a}$ ).

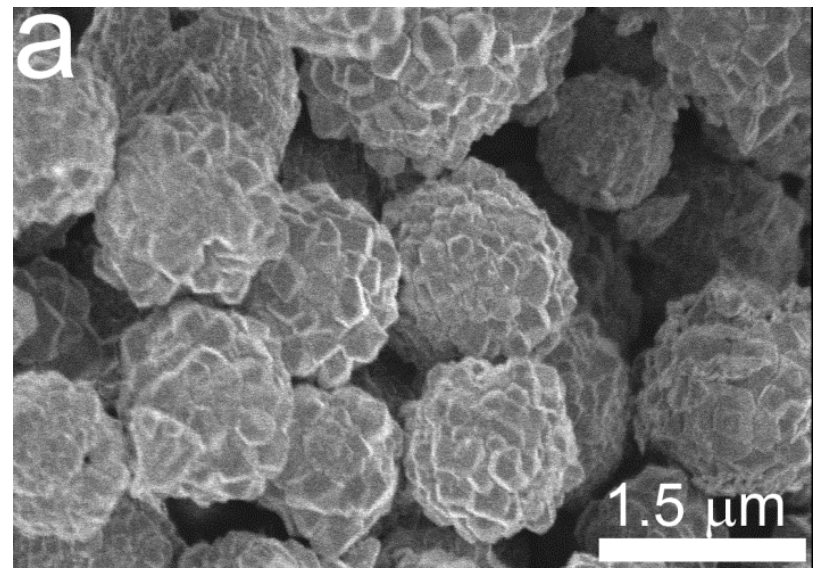

$\mathrm{Cu}$

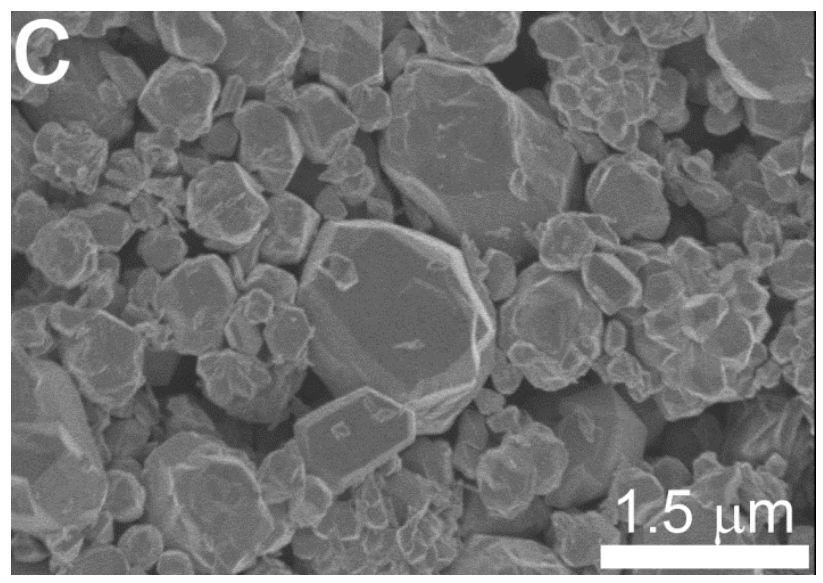

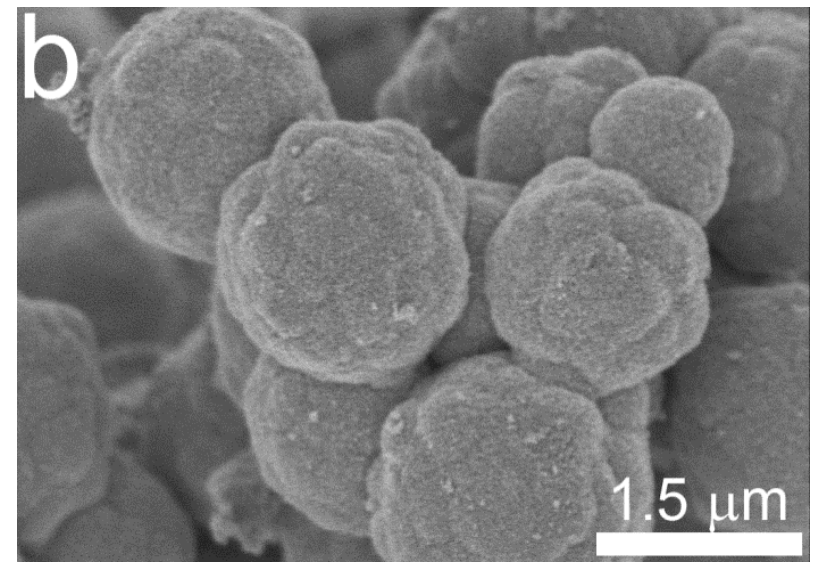

$\mathrm{Pd}$

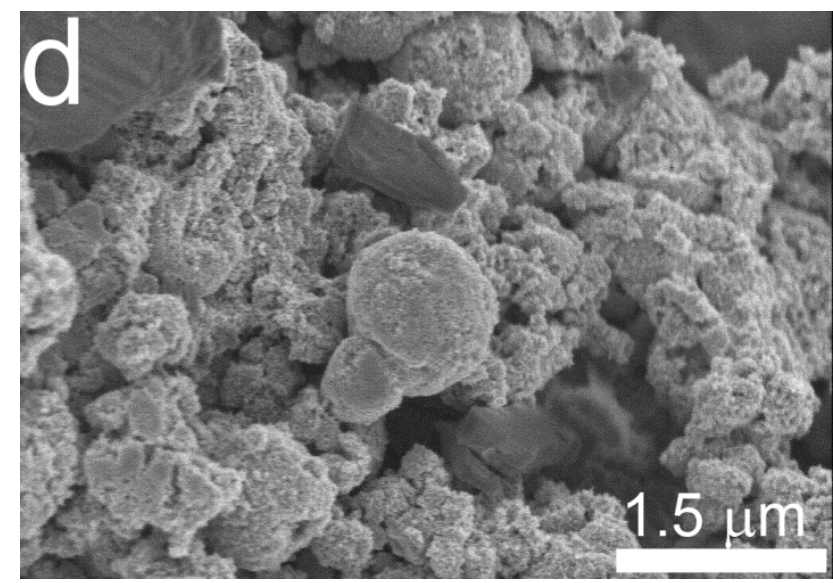

Figure 8: Uniform Cu particles (a) yielded similarly uniform Pd particles (b), while non-uniform $\mathrm{Cu}$ particles (c) yielded Pd with less uniformity (d). Particles were prepared according to the Experimental section, except that surfactant was added within the first two minutes of the $\mathrm{Cu}$ reduction phase, inhibiting the ability of smaller $\mathrm{Cu}$ crystal to aggregate into spherical particles (c).

Variation of several other parameters during the synthesis produced less desirable results. As noted above, preparations using very low or nearly saturated chloride concentrations in the initial reaction solution showed a reduction in overall uniformity of the Pd particles. Additionally, reaction time was observed to be an important factor in producing uniform Pd particles. For reactions lasting longer than 48 hours, there was noticable degradation of particle uniformity. Reaction time also had an effect on the degree to which the copper metal within the reductant particles was oxidized by oxygen. For reactions exposed to oxygen, a green insoluble precipate was observed after 24 hours. This is remedied by bubbling 
nitrogen through the reaction solution to remove oxygen present in the flask. Although nitrogen limits the amount of oxidation during the reaction, it has no effect on particle degradation due to increased reaction time.

The described procedure has been scaled up from an initial product yield of approximately $10 \mathrm{mg}$ to a reaction size yielding approximately $200 \mathrm{mg}$ with negligible changes in results. Because the reactions are dilute, slow, and not extremely exothermic, scaling up is relatively straightforward and requires little modification from the standard procedure. It is expected that further scaling could be easily achieved using larger quantities of reagents.

\subsection{Elemental composition}

Compositional characterization was conducted using EDS in order to determine whether residual copper was present within the Pd particles. Unreacted copper is inert with respect to hydrogen, so it is essentially a diluent in a hydrogen storage material. Residual copper in the form of a palladium alloy can influence the hydrogen storage properties of the product, as previous studies have shown [26]. Residual copper salts such as copper oxide or copper hydroxide are undesirable because they tend to be insoluble, and thus difficult to wash away, and could foreseeably react with hydrogen to form water. Table 1 indicates that particles are composed of $\sim 60 \% \mathrm{Pd}$ and $\sim 40 \% \mathrm{Cu}$, so clearly a significant amount of copper still remains within the particles. In this range of copper content, hydrogen storage properties are significantly diminished [26], so a method to partially remove the copper was developed, as discussed below.

To quantify surface area and measure pore size distribution (PSD), nitrogen porosimetry was performed (Figure 9a). Porosimetry was also conducted on a powder sample of porous Pd after fully hydriding and dehydriding. This demonstrated that the PSD is strongly affected by cycling from the metallic, to the hydrided, and back to the metallic phase. Before cycling, a broad pore size distribution can be seen below $10 \mathrm{~nm}$ with a total surface area of $35 \mathrm{~m}^{2} / \mathrm{g}$. After cycling, the average pore diameter increased, showing a sharp maximum at approximately $10 \mathrm{~nm}$. Furthermore, total surface area is reduced to $22 \mathrm{~m}^{2} / \mathrm{g}$ as the pore diameter increases. 


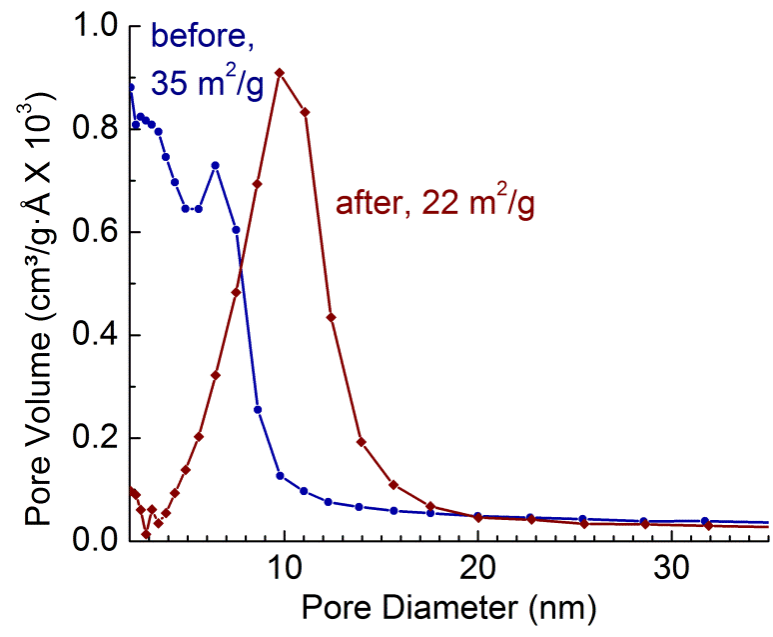

Figure 9: Pore size distribution of reaction product before and after a hydride cycle.

Apparent changes in the pore structure during hydriding and dehydriding cycles may be attributed to the effects of hydrogen when absorbed within the crystal lattice of the metal. Hydrogen stored within the lattice of nanoporous Pd has been demonstrated to have a significant effect on the stability of the pore structure [27]. The change in pore geometry caused by hydrogen cycling collapses smaller pores, causing them to coalesce into large pores, shifting the average pore diameter higher.

\subsection{Hydrogen Storage}

The room-temperature pressure-composition isotherms of the reaction product were measured to determine hydrogen storage capacity, and the pressure range at which hydrogen is absorbed. The samples store an appreciable amount of hydrogen, but do not reach H-to-metal ratios exceeding 0.6 in this pressure range, as is typical for pure $\mathrm{Pd}$ [6]. Rather than exhibiting a plateau at $10 \mathrm{Torr} \mathrm{H}_{2}$ as for pure $\mathrm{Pd}$, the pressure continues to increase until 100 Torr, where only a slight decrease in slope is visible. The lack of a flat plateau region is likely due to a nonuniform copper distribution within a Pd alloy. In a mixture of pure $\mathrm{Cu}$ and pure $\mathrm{Pd}$, the pressure-composition isotherm would resemble that of $\mathrm{Pd}$, except with reduced capacity.

To further distinguish between a mixture and an alloy of $\mathrm{Pd}$ and $\mathrm{Cu}$, XRD analysis was performed on a bulk sample of synthesized Pd powder. Figure 10 compares the collected XRD spectrum to reference peaks for both pure $\mathrm{Pd}$ and $\mathrm{Cu}$ metals. A simulated XRD spectrum for a 30\% $\mathrm{Cu}$-in-Pd alloy was calculated from reference data found in both $\mathrm{Pd}$ and $\mathrm{Cu}$ crystallographic information files using HighScore Plus ${ }^{\circledR}$. The measured peaks are broad, suggesting small grain size or heterogeneity. However, it is clear that the material most closely resembles palladium or the dilute alloy of $\mathrm{Cu}$ in $\mathrm{Pd}$, and there is not a large amount of pure $\mathrm{Cu}$ mixed into the product. The formation of an alloy between a more and less 
noble metal is known to help inhibit the oxidation of the less noble, so it can be expected that alloyed $\mathrm{Cu}$ would not fully react with the Pd salt. If copper atoms are non-uniformly dispersed throughout the Pd, it can be expected that the hydrogen storage properties would be non-uniform throughout the sample.

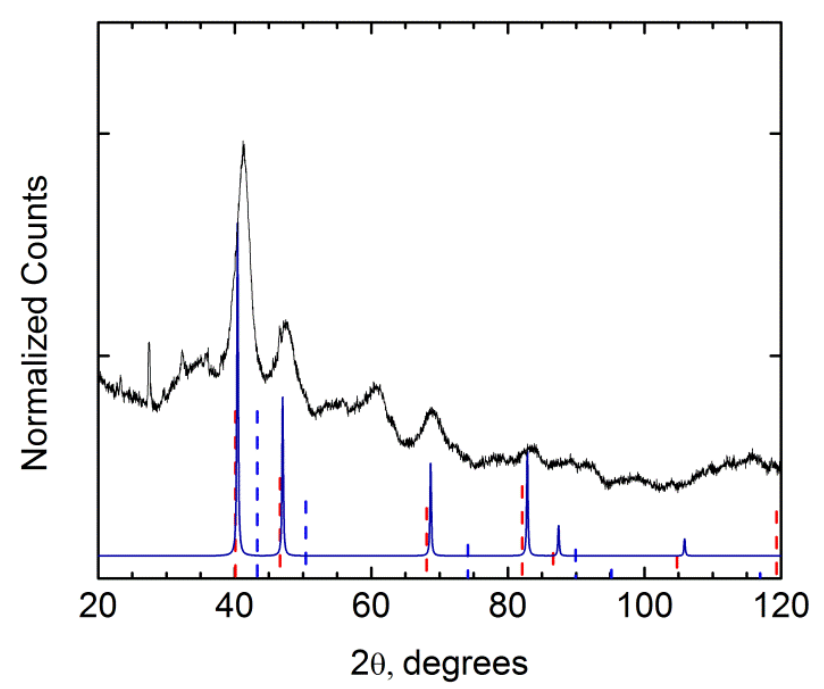

Figure 10: XRD pattern for synthesized particles (grey), compared with calculated spectrum for a Pd-Cu alloy (dark blue), with additional reference peaks for pure $\mathrm{Pd}$ (red) and $\mathrm{Cu}$ (blue).

In an effort to reduce the amount of residual copper within the particles, a chemical treatment with $\mathrm{Pd}\left(\mathrm{NO}_{3}\right)_{2}$ was performed on the Pd alloy. This form of palladium has a higher reduction potential than the $\mathrm{Pd}$ ion in the presence of excess chloride, and can be expected to be more likely to react with residual $\mathrm{Cu}$ in the product that is stabilized by alloying with Pd [28]. Given that average EDS measurements show the Pd particles contain approximately 40 atom \% Cu, a $50 \mathrm{mg}$ sample of bulk Pd powder should contain roughly $14 \mathrm{mg}$ of residual copper. We then exposed the product to a prescribed molar ratio of Pd nitrate to copper.

\begin{tabular}{|c|c|c|c|}
\hline Element & Untreated & $1 \mathrm{Pd}\left(\mathrm{NO}_{3}\right)_{2}: 2 \mathrm{Cu}$ & $2 \mathrm{Pd}\left(\mathrm{NO}_{3}\right)_{2}: 1 \mathrm{Cu}$ \\
\hline $\mathrm{Cu}$ & $38.31 \%$ & $25.66 \%$ & $19.34 \%$ \\
\hline $\mathrm{Pd}$ & $61.69 \%$ & $74.34 \%$ & $80.66 \%$ \\
\hline
\end{tabular}

Table 1. Quantitative EDS analysis of reaction product by atomic \%.

EDS analysis reveals that samples treated with 1:2 and 2:1 ratios of $\mathrm{Pd}\left(\mathrm{NO}_{3}\right)_{2}: \mathrm{Cu}$ show a decrease in $\mathrm{Cu}$ composition. The 1:2 treatment saw a reduction to approximately $25 \% \mathrm{Cu}$, whereas the $2: 1$ treatment saw $\mathrm{Cu}$ composition decrease to $19 \%$ (Table 1). Hydrogen absorption isotherms were recorded for each of the treated samples to determine if the decrease in $\mathrm{Cu}$ composition made any improvement to the hydrogen storage properties of the powder. The resulting isotherms of treated samples are shown in comparison to 
the untreated sample in Figure 11. The results shown are for the second isotherm measured on the sample; the curve was higher in the first measurement, but relatively stable after that. This variation between the first and second measurements may be due to an initial redistribution (most likely a segregation) of copper during the initial hydriding cycle.

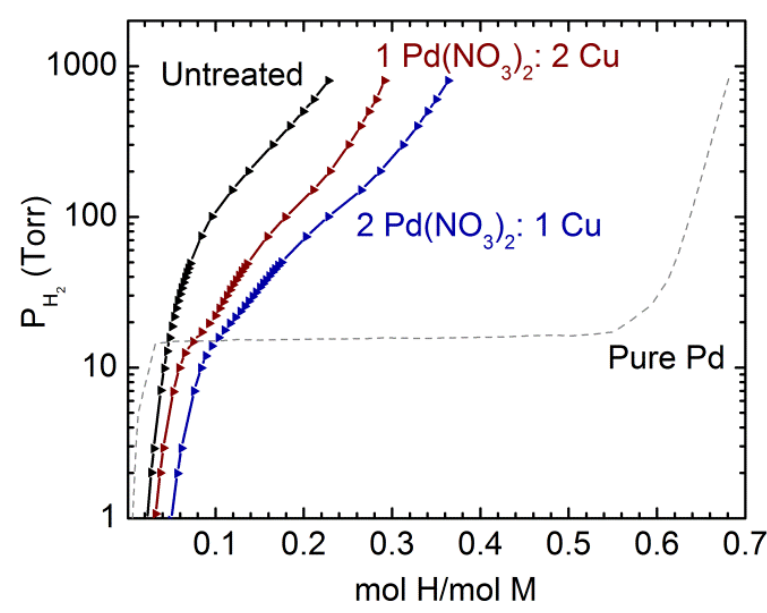

Figure 11: $\mathrm{Pd}-\mathrm{Cu}$ hydrogen absorption isotherms for untreated sample, 1:2 treated sample and 2:1 treated sample. Repeated measurements showed that the first isotherm was different (higher) than subsequent measurements, so the second measurement is shown here.

In pure Pd samples, a pressure plateau at approximately 10 Torr is visible. Although a clear plateau is not visible in the treated samples, there is a noticeable decrease in slope at the 10 Torr mark, suggesting a higher Pd composition. The residual copper remaining in the treated metal is unlikely to be uniformly distributed, and thus still limits the absorption and prevents a plateau. Even though copper is still present within the powder, the treatment process produces a noticeable increases in the hydrogen absorption capacity at both high and low pressures. Hydrogen absorption at pressures below 10 Torr can be attributed to surface adsorption, because the surface hydride is more stable than the bulk hydride [29]. The increased amount of hydrogen absorbed at the lowest pressures shown may be due to an increase in the amount of pure $\mathrm{Pd}$ at the surface as the $\mathrm{Cu}$ near the surface is removed.

\section{Conclusions}

Through the self-limiting reaction between Pd salts and metallic copper particles in the presence of a surfactant, we have developed a straightforward, inexpensive procedure to produce uniform nanoporous Pd alloy particles. This procedure should produce size- and shape-tunable particles based on the physical characteristics of the copper used as a reducing agent. This approach cannot be expected to succeed if the 
copper particles or growing intermediates aggregate, or if many small Pd particles grow and break off of the $\mathrm{Cu}$. We were able to identify reaction conditions that avoid such dead ends, yielding consistently reproducible results.

Our experience suggests that these results are scalable to even greater quantities. Even though further study may be necessary to optimize the reduction processes, this method offers a practical approach to a palladium alloy with uniform pore and particle size. Despite the fact that residual $\mathrm{Cu}$ within the powder may reduce the overall absorption capabilities, a $\mathrm{Pd}-\mathrm{Cu}$ alloy may be beneficial in specific applications. $\mathrm{Pd}-\mathrm{Cu}$ alloys have application in such areas such as hydrogen gas and isotope separation, where achievement of maximum possible storage densities is not necessary. Potential applications in other fields, such as medical devices or chemical separations, may also be possible in the future [30].

\section{Acknowledgements}

The authors thank Jeffrey Chames and Charles Steinhaus for assistance with SEM. Sandia National Laboratories is a multi-program laboratory managed and operated by Sandia Corporation, a wholly owned subsidiary of Lockheed Martin Corporation, for the U.S. Department of Energy's National Nuclear Security Administration under contract DE-AC04-94AL85000.

\section{References}

[1] A.F. Burke, Batteries and Ultracapacitors for Electric, Hybrid, and Fuel Cell Vehicles, Proc. IEEE 95 (2007) 806-820.

[2] J.W. Long, B. Dunn, D. R. Rolison, H. S. White, Three-Dimensional Battery Architectures, Chem. Rev. 104 (2004) 4463-4492.

[3] D.B. Robinson, Optimization of Power and Energy Densities in Supercapacitors, J. Power Sources 195 (2009) 3748-3756.

[4] P.J. Cappillino , K.M. Hattar , B.G. Clark , R.J. Hartnett, V. Stavila , M.A. Hekmaty , B. W. Jacobs, D. B. Robinson, Synthesis of mesoporous palladium with tunable porosity and demonstration of its thermal stability by in situ heating and environmental transmission electron microscopy, J. Mater. Chem. A. 1 (2013) 602-610. 
[5] F.A. Lewis, The Hydrides of Palladium and Palladium Alloys, Platinum Metal Reviews 4 (1960)132137.

[6] T.B. Flanagan, W.A. Oates, The Palladium-Hydrogen System, Ann. Rev. Mater. Sci. 21 (1991) 269304.

[7] G.S. Attard, P.N. Bartlett, N R.B. Coleman, J.M. Elliott, J.R. Owen, J.H. Wang, Mesoporous Platinum Films from Lyotropic Liquid Crystalline Phases, Science 278 (1997) 838-840.

[8] P.N. Bartlett, B. Gollas, S. Guerin, J. Marwan, The preparation and characterisation of $\mathrm{H}_{1}$-e palladium films with a regular hexagonal nanostructure formed by electrochemical deposition from lyotropic liquid crystalline phases, Phys. Chem. Chem. Phys. 4 (2002) 3835-3842.

[9] D.B. Robinson, S.J. Fares, M.D. Ong, I. Arslan, M.E. Langham, K.L. Tran, W.M. Clift, Scalable synthesis of nanoporous palladium powders, Int. J. Hydrogen Energy 34 (2009) 5585-5591.

[10] D.B. Robinson, M.E. Langham, S.J. Fares, M.D. Ong, B.W. Jacobs, W.M. Clift, J.K. Murton, R.P. Hjelm, M.S. Kent, Thermally stable nanoporous palladium alloy powders by hydrogen reduction in surfactant templates, Int. J. Hydrogen Energy 35 (2010) 5423-5433.

[11] W. Sugimoto, S. Makino, R. Mukai, Y. Tatsumi, K. Fukuda, Y. Takasu, Y. Yamauchi, Synthesis of ordered mesoporous ruthenium by lyotropic liquid crystals and its electrochemical conversion to mesoporous ruthenium oxide with high surface area, J. Power Sources 204 (2012) 244-248.

[12] W.S. Chae, D. Van Gough, S.K. Ham, D.B. Robinson, P.V. Braun, Effect of Ordered Intermediate Porosity on Ion Transport in Hierarchically Nanoporous Electrodes, ACS Appl. Mater. Interf. 4 (2012) 3973-3979.

[13] C. Ji, P.C. Searson, Synthesis and Characterization of Nanoporous Gold Nanowires, J. Phys. Chem. B 107 (2003) 4494-4499.

[14] V. Badri, A.M. Hermann, Metal hydride batteries: Pd nanotube incorporation into the negative electrode, Int. J. Hydrogen Energy 25 (2000) 249-253 
[15] X.F. Lu, M. McKiernan, Z.M. Peng, E.P. Lee, H. Yang, Y.N. Xia, Noble-Metal Nanotubes Prepared via a Galvanic Replacement Reaction between $\mathrm{Cu}$ Nanowires and Aqueous $\mathrm{HauCl}_{4}, \mathrm{H}_{2} \mathrm{PtCl}_{6}$, or $\mathrm{Na}_{2} \mathrm{PdCl}_{4}$, Sci. Adv. Mater. 2 (2010) 413-420.

[16] L.B. Sheridan, D. K. Gebregziabiher, J.L. Stickney, D.B. Robinson, Formation of Palladium Nanofilms Using Electrochemical Atomic Layer Deposition (E-ALD) with Chloride Complexation, Langmuir 29 (2013) 1592-1600.

[17] H. Wang, L. Wang, T. Sato, Y. Sakamoto, S. Tominaka, K. Miyasaka, N. Miyamoto, Y. Nemoto, O. Terasaki, Y. Yamauchi, Synthesis of Mesoporous Pt Films with Tunable Pore Sizes from Aqueous Surfactant Solutions, Chem. Mater. 24 (2012) 1591-1598.

[19] S. Lowell, J.E. Shields, M.A. Thomas, M. Thommes, Characterization of Porous Solids and Powders: Surface Area, Pore Size and Density, Kluwer Academic Publishers, Dordrecht, NL, 2004.

[20] E.P. Barrett, L.G. Joyner, P P. Halenda, J. Am. Chem. Soc. 73 (1951) 373-380.

[21] M. Muzikář, P. Polášková, J.C. Fettinger, W.R. Fawcett, Electrochemical Growth of Platinum Particles and Platinum-Containing Crystals in Silica Gel, Crystal Growth \& Design 6 (2006) 1956-1960.

[22] H.P. Cong, S.H. Yu, Shape Control of Cobalt Carbonate Particles by a Hydrothermal Process in a Mixed Solvent: An Efficient Precursor to Nanoporous Cobalt Oxide Architectures and Their Sensing Property, Crystal Growth \& Design 9 (2009) 210-217.

[23] A. Aimable, A.T. Puentes, P. Bowen, Synthesis of porous and nanostructured particles of CuO via a copper oxalate route, Powder Tech. (2011) 467-471.

[24] M. Biçer, İ. Şişman, Controlled synthesis of copper nano/microstructures using ascorbic acid in aqueous CTAB solution, Powder Tech. 198 (2010) 279-284. 
[25] G. Wanka, H. Hoffmann, W. Ulbricht, Phase diagrams and aggregation behavior of poly(oxyethylene)-poly(oxypropylene)-poly(oxyethylene) triblock copolymers in aqueous solutions, Macromolecules 27 (1994) 4145-4159.

[26] R. Burch, R.G. Buss, Absorption of Hydrogen by Palladium-Copper Alloys, J. Chem. Soc. Faraday Trans. 71 (1975) 913-921.

[27] M.D. Ong, B.W. Jacobs, J.D. Sugar, M.E. Grass, Z. Liu, G.M. Buffleben, W.M. Clift, M.E. Langham, P.J. Cappillino, D.B. Robinson, Effect of Rhodium Distribution on Thermal Stability of Nanoporous Palladium-Rhodium Powders, Chem. Mater. 24 (2012) 996-1004.

[28] A.J. Bard, R. Parsons, J. Jordan, Standard Potentials in Aqueous Solution, CRC Press, New York, 1985.

[29] P.J. Cappillino, M.A. Hekmaty, B.W. Jacobs, J.D. Sugar, V. Stavila, P.G. Kotula, J.M. Chames, N.Y. Yang, D.B. Robinson, Nanoporous Pd Alloys with Compositionally Tunable Hydrogen Storage Properties Prepared by Nanoparticle Consolidation, Journal of Materials Chemistry 22 (2012) 1410314022.

[30] S.D. Gittard, B.E. Pierson, C.M. Ha, C.A.M. Wu, R.J. Narayan, D.B. Robinson, Supercapacitive transport of pharmacologic agents using nanoporous gold electrodes, Biotech. J. 5 (2010) 192-200. 

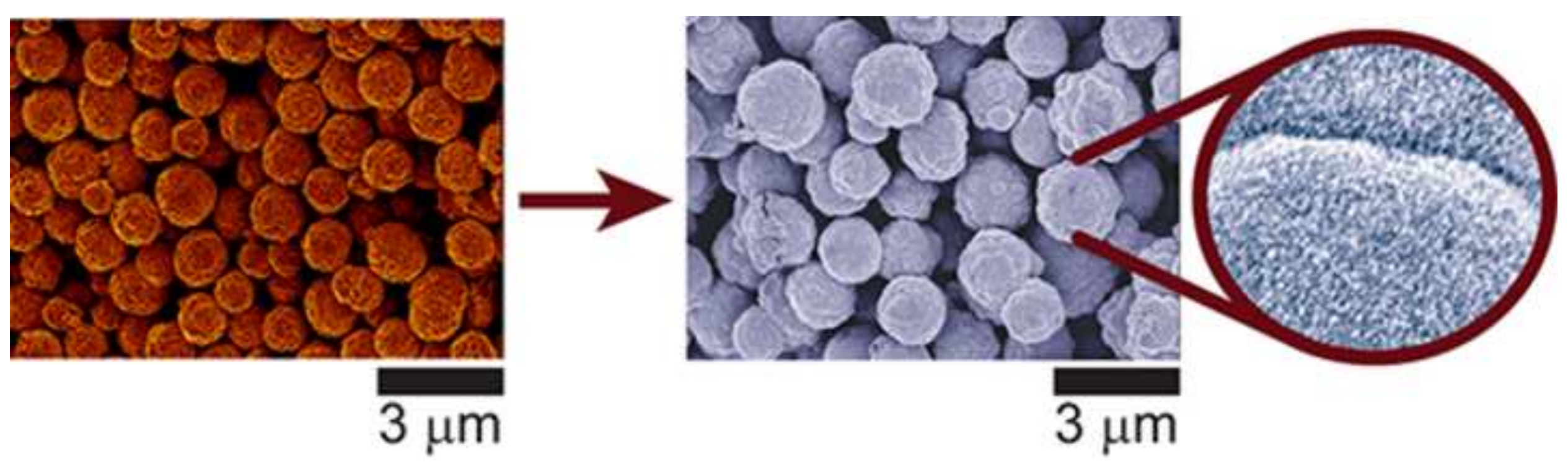

.

Graphical Abstrac
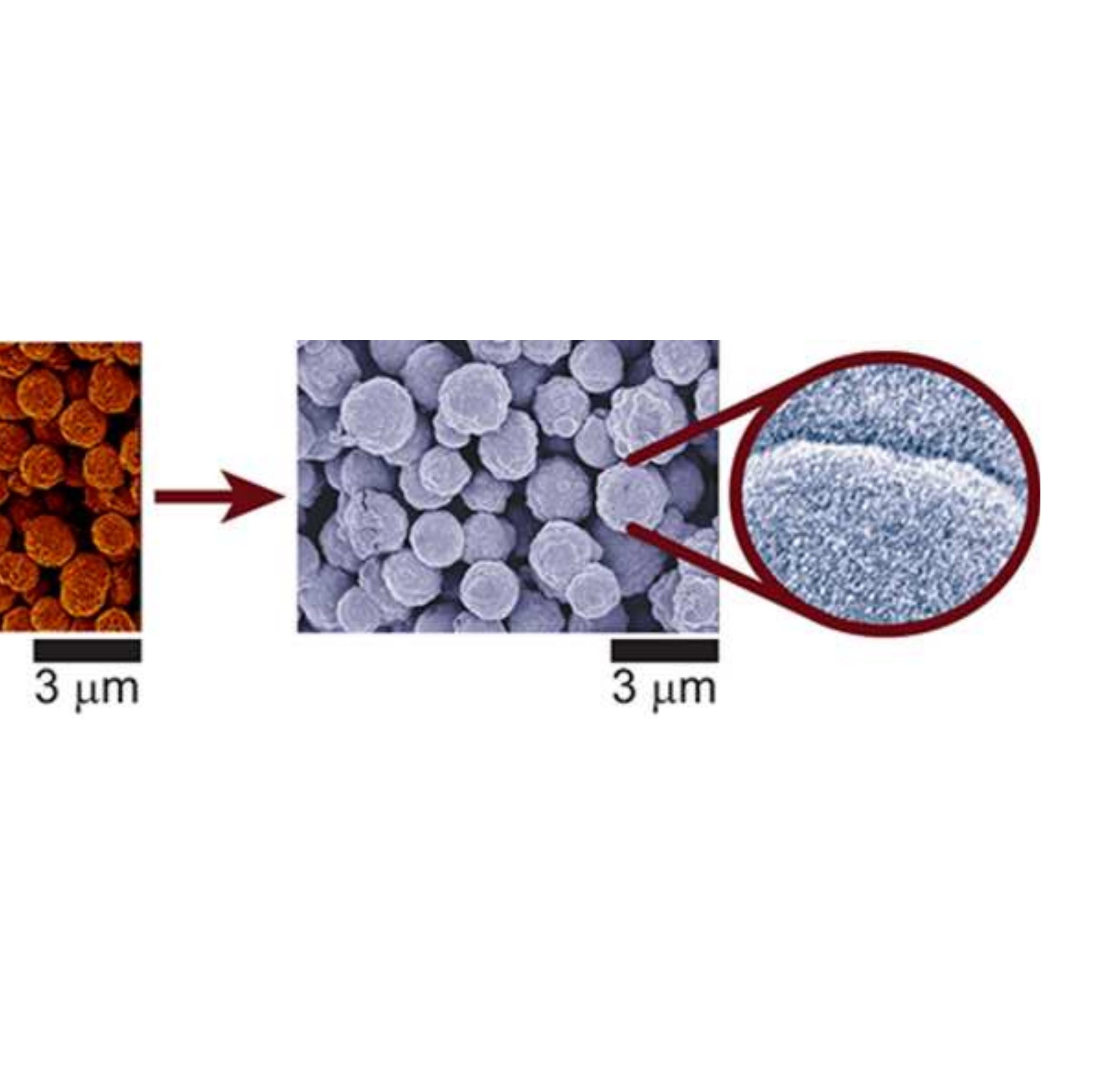